\title{
Double primary lung adenocarcinoma diagnosed by epidermal growth factor receptor mutation status
}

\author{
Oh Jung Kwon', Min Hyeok Lee', Sung Ju Kang ${ }^{1}$, Seul Gi Kim¹, In Beom Jeong ${ }^{1}$, Ji Yun Jeong ${ }^{2}$, \\ Eun Jung $\mathrm{Cha}^{3}$, Do Yeun Cho', Young Jin $\mathrm{Kim}^{4}$, Ji Woong Son ${ }^{1}$ \\ ${ }^{I}$ Department of Internal Medicine, Konyang University Hospital, Daejeon; ${ }^{2}$ Department of Pathology, \\ Kyungpook National University Medical Center, Kyungpook National University School of Medicine, Daegu; \\ Departments of ${ }^{3}$ Pathology and ${ }^{4}$ Thoracic Surgery, Konyang University Hospital, Daejeon, Korea
}

\begin{abstract}
A nodular density was detected on a chest radiograph taken from a 57-year-old Korean woman who was visiting a hospital for a routine check. Chest computed tomography revealed a $4.8 \mathrm{~cm}$ lobulated mass in the right lung and another focal nodular lesion in the left lung; biopsies of both lungs revealed adenocarcinoma. We conducted DNA sequencing and peptide nucleic acid clamping to investigate the potential double primary lung cancer. The results verified that the mass in the right lung had a mutation in the epidermal growth factor receptor, whereas the nodule in the left lung had a wild-type sequence, showing that these two were genetically different cancers from one another. Thus, we demonstrate that genetic testing is useful in determining double primary lung cancer, and we herein report on this case.
\end{abstract}

Keywords: Adenocarcinoma; Differential diagnosis; Lung cancer; DNA sequence analysis; Mutations

\section{INTRODUCTION}

The number of patients with multiple primary lung cancer (MPLC) is increasing [1]. The concept of MPLC was introduced in 1924 [2], and second primary tumors are either metachronous - being detected after the primary lesion, or synchronous - being resected or detected simultaneously. Synchronous MPLC was presumed to be uncommon; however, in reality its true incidence, which ranges from $0.2 \%$ to $20 \%$ [3], is increasing owing to the wide usage of positron emission tomography (PET) scanning and improvements in computed tomography (CT) scanning and other diagnostic modalities.

Received: July 11, 2016, Revised: September 13, 2016 Accepted: September 26, 2016

Corresponding Author: Ji Woong Son, Deprartment of Internal Medicine, Konyang University Hospital, Gwanjeo dong-ro 158, Seo-gu, Daejeon 35365, Korea Tel: +82-42-600-8817, Fax: +82-42-600-9090

E-mail: sk1609@hanmail.net
When multiple synchronous lung tumors have been identified, the differentiation of multi-centric cancers from single cancers with pulmonary metastases or intrapulmonary metastases from primary cancers in different organs can often be difficult, especially if the histologic characteristics of synchronous tumors are the same.

We present a case of synchronous double primary lung cancers; an adenocarcinoma with an epidermal growth factor receptor (EGFR) mutation in the right lung and an adenocarcinoma without mutation in the left lung.

\section{CASE}

A 57-year-old Korean woman visited a hospital for a routine check-up. A nodular density was detected on a chest radiography. She was healthy with no symptoms and had an unremarkable family or medical history. In addition, she had no history of smoking. Her vital signs revealed a blood pressure of $110 / 71 \mathrm{mmHg}$, a heart rate of 81 beats per mi-

Copyright (C) 2017 Yeungnam University College of Medicine

This is an Open Access article distributed under the terms of the Creative Commons Attribution Non-Commercial License (http://creativecommons.org/licenses/by-nc/4.0/) which permits unrestricted non-commercial use, distribution, and reproduction in any medium, provided the original work is properly cited. 
nute, and a respiration rate of 17 breaths per minute; her laboratory findings were all normal. Her general condition was good, and a physical examination showed no abnormalities. Chest X-ray showed an abnormal shadow in the right middle lung field. A chest CT scan revealed a $4.8 \mathrm{~cm}$ lobulated mass in the right upper lobe (RS3) and another focal nodular lesion in the left upper lobe (LS3) (Fig. 1). We observed no mediastinal lymph node enlargement and noted no metastatic lesions on brain magnetic resonance imaging san, PET-CT scan, or whole-body bone scan. The levels of serum tumor markers, such as carcinoembryonic antigen, cytokeratin fragment, and neuron-specific enolase, were within normal range.
We performed two percutaneous needle aspiration biopsies (NABs) for each of the nodules and collected biopsy specimens from both lesions. There were no complications during $\mathrm{NAB}$, and the patient had an uneventful recovery. Histological examinations of both specimens revealed adenocarcinomas in both lungs (Fig. 2).

To determine whether the lesions arose independently or as a result of metastasis, we conducted direct sequencing of the deoxyribonucleic acid (DNA) from both tumors. Exon sequencing of the genomic DNA revealed deletion and substitution mutations of EGFR in the right lung nodule and a wild-type sequence in the left lung nodule (Fig. 3). Given the
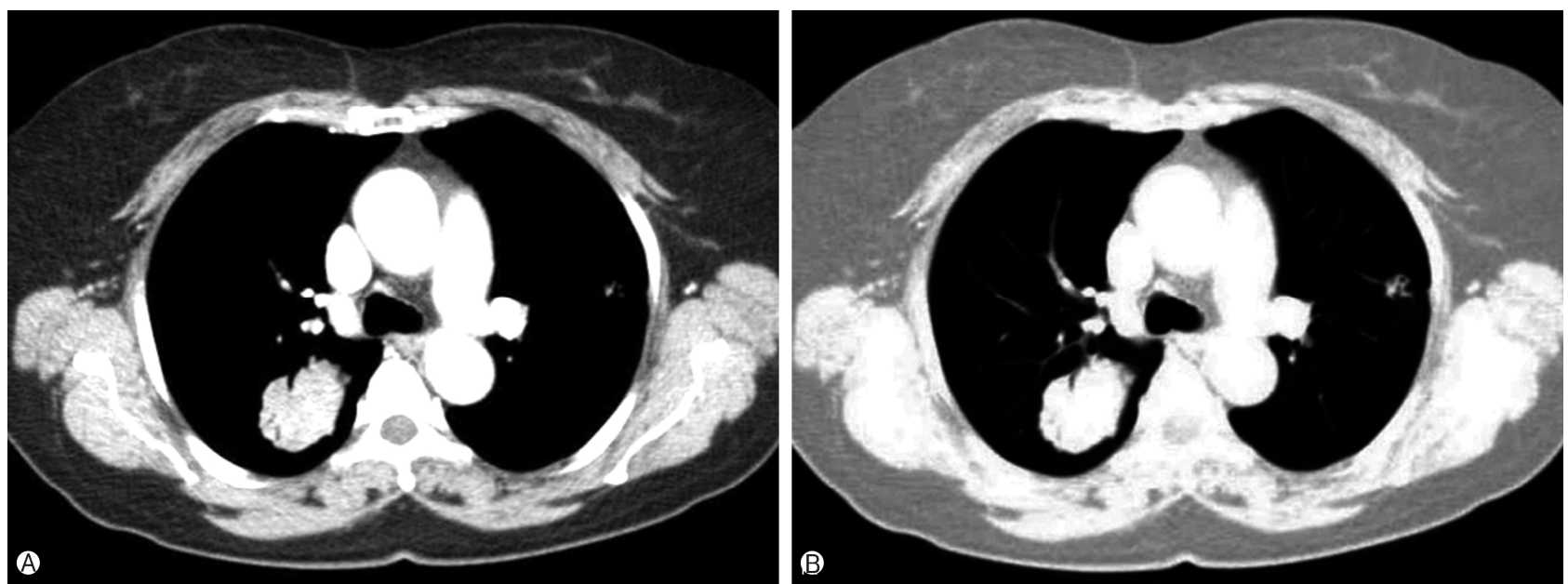

Fig. 1. Computed tomography scan showing a $4.8 \mathrm{~cm}$ lobulated mass with air bronchogram in the right posterior segment (A). Another focal nodular lesion is evident in the left anterior segment with a lucent internal bubble (B).

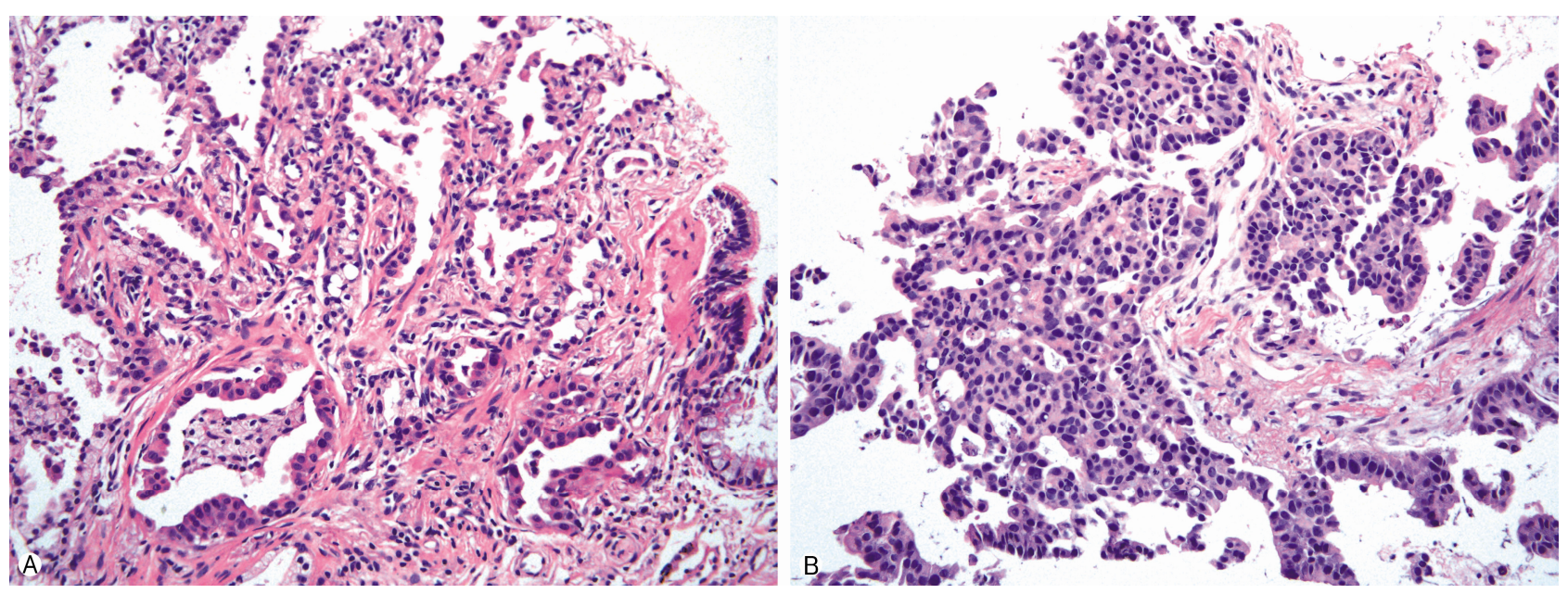

Fig. 2. The left lung adenocarcinoma shows an acinar and lepidic pattern (A), while adenocarcinoma in the right lung consists of tumor cell nests with poorly-formed and small glandular spaces (B). (A, B, H\&E stain, ×200). 
(A) delL747-P753insS (sense)
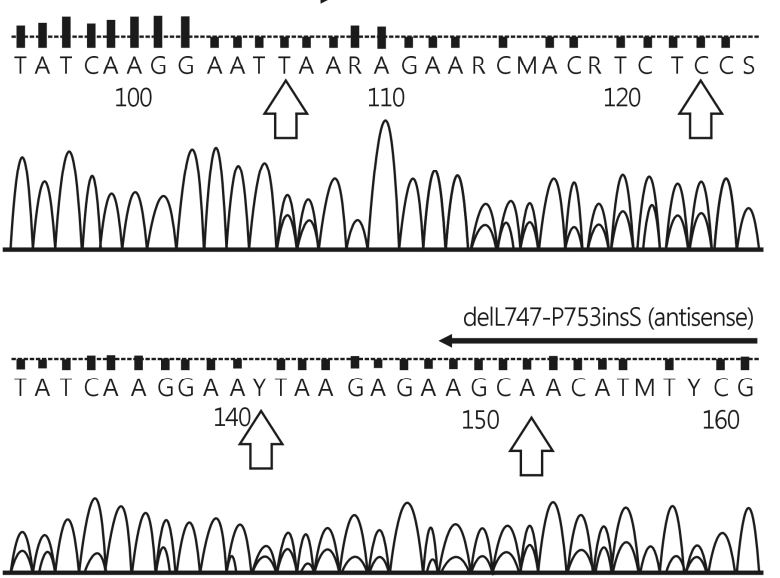

(B)

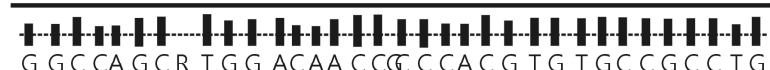

80

90

100

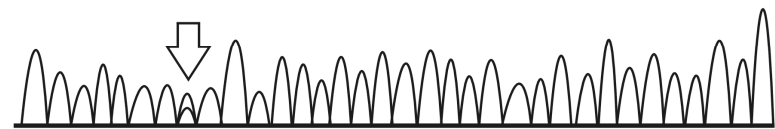

Fig. 3. Direct sequencing DNA demonstrating an exon 19 deletion (A) and an exon 20 substitution (B) in the tumor in the right lung.

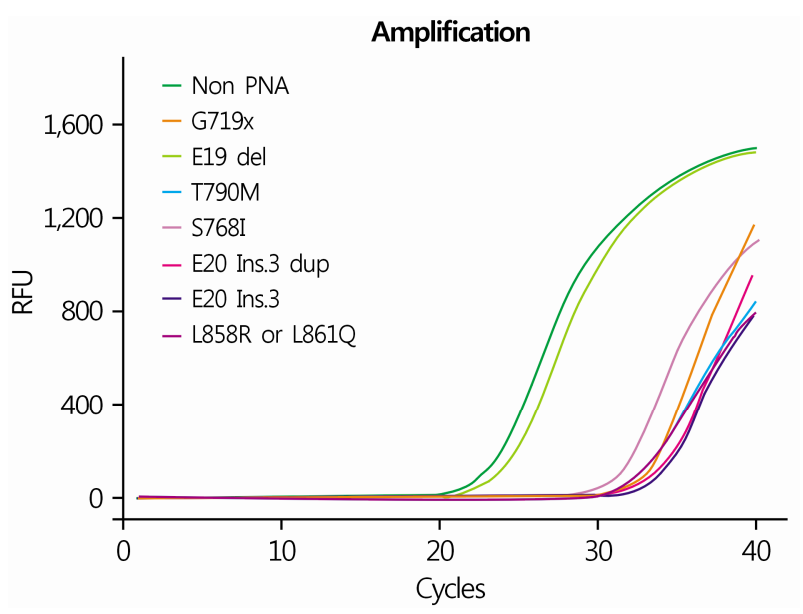

Fig. 4. PNA-mediated real-time PCR clamping. A PNA/DNA hybrid with a single base pair mismatch did not suppress annealing of the PCR primer or amplification of mutant alleles (exon 19 deletion). PNA, peptide nucleic acid; PCR, polymerase chain reaction; RFU, relative fluorescence unit.

low sensitivity of direct sequencing, especially for a small mass, a peptide nucleic acid (PNA)-mediated real-time polymerase chain reaction (PCR) clamping was performed, which revealed a positive signal in exon 19 (deletion) from the sample of the right lung (Fig. 4) and no mutation in the left lung (data not shown). Although the NAB test was not able to represent the overall characteristics of the lesion due to the small size of the sample, additional highly-specific DNA analysis and highly-sensitive PNA test provided reliability to the results.

We performed a right upper lobectomy in March 2014 and a left upper lobe anterior and superior lingular segmentec- tomy in April, 2014. There were no intraoperative complications, and the patient fully recovered. Pathologic staging of the nodule in the right lung was pT2aN2 (stage IIIA), whereas that in the left lung was pT2aN0 (stage IB). Both nodules were positive for thyroid transcription factor-1. Adjuvant concurrent chemoradiation therapy was administered after the surgery.

During the follow-up period, there was recurrence only in the right-side (lower right lung, RS6). After the treatment with an EGFR tyrosine kinase inhibitor, a partial response state was maintained.

\section{DISCUSSION}

Lung cancer can be divided into four main types: squamous cell carcinoma, small cell carcinoma, large cell carcinoma, and adenocarcinoma. Especially in adenocarcinoma, a more precise subtyping is made available due to the molecular analytic techniques. According to the presence or absence of a driving mutation, the clinical characteristics and optimal treatments are different. Therefore, genetic analysis of the driving mutation is an essential tool in lung cancer diagnosis and treatment.

The diagnosis of a second primary lung cancer is complex for a number of reasons. It can be difficult to differentiate a metastasis from the primary lesion, if the latter occurs within 2 years of the initial tumor. Differentiation is also hindered when the initial tumor and second primary lesion have a similar histological subtype and when the second lesion is located in an area previously exposed to radiotherapy. This is the 
case because changes in tissue morphology can complicate the differential diagnosis.

Direct sequencing and PNA clamping are approved by the Korean Food and Drug Administration. A PNA is an artificially synthesized polymer that can bind to a complementary sequence in DNA; the binding capacity of PNA is stronger than that of DNA. PNA clamping can detect 29 target mutations of clinical significance among approximately 250 known EGFR mutations [4], but it cannot detect unknown EGFR mutation sites. The weakness of PNA clamping is that this method can only be used to detect mutations for which the primers have been individually designed; conversely, direct sequencing can uncover novel mutations. In this case, PNA clamping did not detect substitution mutations in exon 20 .

The diagnosis is complicated by difficulties in clearly making a distinction between MPLC and metastatic lung cancer. Clinicopathologic diagnosis, assessment, and management have all evolved, but still remain severely limited by a lack of dependable and robust molecular markers for the differential diagnosis of MPLC and metastasis. The Martini and Melamed clinicopathological assessment criteria are based on tumor characteristics that include, but are not limited to, the location, morphology, absence or presence of carcinoma in situ, metastasis, and vascular invasion [5]. However, these criteria lack the power to differentiate between a second primary lung cancer and metastasis [6,7]. Genomic analysis of patients is useful to distinguish between a second primary lung cancer and metastasis. Girard et al. found that genomic profiling contradicted the clinicopathological diagnosis in $18 \%$ of tumors [8]. That same group reported that EGFR and K-ras mutation status could be used to determine the existence of clonal relationships between MPLCs. EGFR/K-ras mutational profiling was useful in improving the discrimination between intrapulmonary metastases and MPLC. In a search of the literature, we found four cases with simultaneous EGFR mutations in multiple lesions. Two cases had the same mutation, while the other two cases had different mutations. In the case of simultaneous EGFR mutations, examination of additional somatic tumor gene alterations by next generation sequencing is useful in differentiating between metastasis and primary lung cancer [9]. However, next generation sequencing may be limited as it is very expensive and is rarely used in clinical settings. Developing rapid, inexpensive, and reliable molecular tools may allow the refinement of the clini- copathologic criteria that are currently used in MPLCs [10].

Precise staging and an aggressive surgical approach that includes tissue-sparing procedures for early-stage cancers should be adapted as the standard clinical practice to increase longterm survival and maintain respiratory function in patients with MPLC. The present case used direct sequencing and PCR clamping to diagnose primary adenocarcinomas in both lungs based on the EGFR mutation status. Genetic analysis presents an extremely attractive method to diagnose double primary cancer.

\section{CONFLICT OF INTEREST}

No potential conflict of interest relevant to this article was reported.

\section{REFERENCES}

1. Xue X, Liu Y, Pan L, Wang Y, Wang K, Zhang M, et al. Diagnosis of multiple primary lung cancer: a systematic review. J Int Med Res 2013;41:1779-87.

2. Beyreuther H. Multiplicität von Carcinomen bei einem Fall von sog. "Schneeberger" Lungenkrebs mit Tuberkulose. Virchows Arch Path Anat 1924;250:230-43.

3. Rea F, Zuin A, Callegaro D, Bortolotti L, Guanella G, Sartori F. Surgical results for multiple primary lung cancers. Eur J Cardiothorac Surg 2001;20:489-95.

4. Lee HJ, Xu X, Kim H, Jin Y, Sun P, Kim JE, et al. Comparison of direct sequencing, PNA clamping-real time polymerase chain reaction, and pyrosequencing methods for the detection of EGFR mutations in non-small cell lung carcino$\mathrm{ma}$ and the correlation with clinical responses to EGFR tyrosine kinase inhibitor treatment. Korean J Pathol 2013;47: 52-60.

5. Martini N, Melamed MR. Multiple primary lung cancers. J Thorac Cardiovasc Surg 1975;70:606-12.

6. Chang YL, Wu CT, Lee YC. Surgical treatment of synchronous multiple primary lung cancers: experience of 92 patients. J Thorac Cardiovasc Surg 2007;134:630-7.

7. Ostrovnaya I, Olshen AB, Seshan VE, Orlow I, Albertson DG, Begg CB. A metastasis or a second independent cancer? Evaluating the clonal origin of tumors using array copy number data. Stat Med 2010;29:1608-21.

8. Girard N, Ostrovnaya I, Lau C, Park B, Ladanyi M, Finley $\mathrm{D}$, et al. Genomic and mutational profiling to assess clonal relationships between multiple non-small cell lung cancers. Clin Cancer Res 2009;15:5184-90.

9. Chuang JC, Shrager JB, Wakelee HA, Neal JW. Concordant and discordant EGFR mutations in patients with multifocal adenocarcinomas: implications for EGFR-targeted therapy. Clin Ther 2016;38:1567-76. 
On Jung Kwon et al.

10. Girard N, Deshpande C, Azzoli CG, Rusch VW, Travis WD, Ladanyi $\mathrm{M}$, et al. Use of epidermal growth factor receptor/ Kirsten rat sarcoma 2 viral oncogene homolog mutation test- ing to define clonal relationships among multiple lung adenocarcinomas: comparison with clinical guidelines. Chest 2010; $137: 46-52$. 